\title{
O USO DE POKA-YOKE NO PROCESSO DE PRESCRIÇÃO MÉDICA DE UMA CLÍNICA DE DOENÇAS NEOPLÁSICAS ${ }^{1}$
}

\section{THE USE OF POKA-YOKE IN THE MEDICAL PRESCRIPTION PROCESS IN A NEOPLASIC DISEASES CLINIC}

\section{EL USO DE POKA-YOKE EN EL PROCESSO DE PRESCRIPCIÓN MÉDICA DE UNA CLÍNICA DE ENFERMEDAD NEOOPLASIA}

\author{
REGINA CÉLIA NAZAR FIALHO \\ Universidade Federal de Minas Gerais \\ reginanfialho@gmail.com
}

NOEL TORRES JÚNIOR

Universidade Federal de Minas Gerais noelface@gmail.com

\author{
CIBELE MARIA VITARELLI ANTUNES DA SILVA \\ Oncomed \\ cibele@ oncomedbh.com.br
}

LILIAN CUNHA LAGE

Oncomed

Lilian@ oncomedbh.com.br

\begin{abstract}
RESUMO
Este trabalho tem como objetivo compreender como mecanismos à prova de falha podem influenciar a qualidade percebida de clientes de uma clínica de doenças neoplásicas. Trata-se de uma pesquisa qualitativa, um estudo descritivo, que se apoia em um estudo de caso do processo de prescrição médica de uma clínica especializada na prevenção e no tratamento de câncer. A partir dos dados levantados, foi possível constatar, através de ferramentas estatísticas, que falhas são inevitáveis em serviços de saúde e que o uso de poka-yokes possibilita uma melhoria nos índices de satisfação relacionados a confiabilidade e dimensões interpessoais do serviço. Sendo assim, o uso de dispositivos a prova de falha melhoram a percepção da qualidade do serviço de saúde prestado.
\end{abstract}

PALAVRAS-CHAVE: Erros Médicos; Poka-Yokes; Qualidade em serviços de saúde;

\begin{abstract}
This work aims at understanding how mistake-proof mechanisms can influence clients's perceived quality of a neoplasic diseases clinic. It consists of qualitative research, a descriptive study, which anchors itself on a case study regarding the administration of medicines in a clinic specialized in the prevention and treatment of cancer. Using the gathered data, it was possible to ascertain, through statistics tools, that mistakes are inevitable in health services and also that the use of poka-yoke promotes an increase in satisfaction when it comes to dependability and the interpersonal dimension of the service. Therefore, the use of mistake-proof devices fosters a more positive and satisfactory view of the health service rendered.
\end{abstract}

KEYWORDS: Medical Errors; Poka-Yokes; Health Services Quality;

\section{RESUMEN}

Este trabajo señala hacia el entendimiento de cómo los mecanismos relacionados a la fiabilidad pueden influenciar la calidad percibida por el cliente de una clínica de enfermedad neoplasia. Esta consiste de una investigación de calidad, un estudio descriptivo que se ancla a un caso de estudio con respecto a la administración de medicinas en una clínica especializada en la prevención y tratamiento de cáncer. Usando la información adquirida fue posible acertar que los errores son inevitables en servicios de salud como también en comprobaciones, y confirmar que el uso de poka-yokes promueve una mejora de los índices de satisfacción con

\footnotetext{
${ }^{1}$ Submetido em 08 de agosto de 2013. Aceito em 14 de fevereiro de 2014. O artigo foi avaliado segundo o processo de duplo anonimato e avaliado pelo editor. Editores responsáveis: Márcio Augusto Gonçalves e Lucas Maia dos Santos. Reprodução parcial ou total e trabalhos derivativos permitidos com a citação apropriada da fonte.
} 

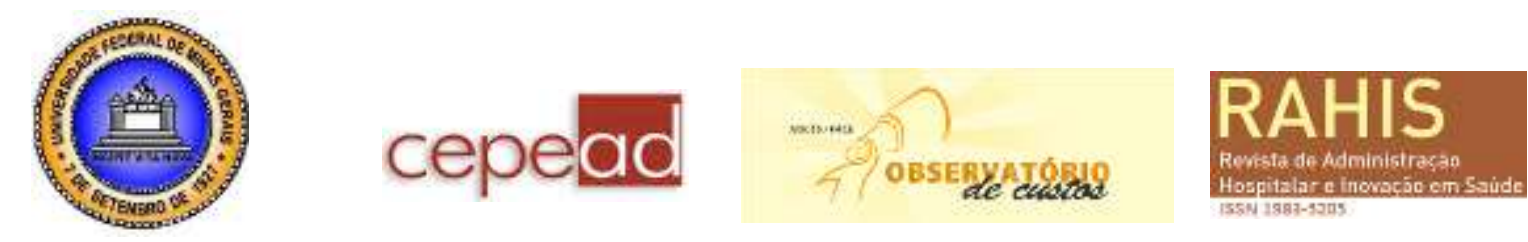

dimensiones interpersonales del servicio. Los resultados del estudio indican la importancia de su uso en aparatos libres de errores con servicios de salud.

PALABRAS-CLAVE: Errores Médicos; Poka-Yoke; Calidad en Servicios de Salud;

\section{Introdução}

Considerando a grande complexidade da atividade médica e de profissionais de saúde e o grande número de intervenções que um paciente está sujeito, existe um grande risco de incidências de erros em um processo de atendimento a pacientes. Os erros em serviços de saúde acontecem em uma rede de relações e podem gerar impactos irreversíveis para o paciente (COLI; ANJOS; PEREIRA, 2010).

Segundo recentes estudos, um a cada dez pacientes atendidos em hospitais europeus sofre danos que poderiam ser evitados. Nos Estados Unidos essa estimativa é de um a cada três pacientes (O’CONNOR et al.,2010; STEENHUYSEN, 2011). Diante destas estatísticas, a afirmação de Spear (2005) que "você está tão seguro em um hospital como você estaria ao fazer pára-quedismo de uma ponte ou um prédio" faz sentido.

Uma resposta a esta realidade passa pela melhoria de operações internas. Novas abordagens de gestão, largamente utilizadas nas indústrias, como Sistema Toyota de Produção podem ser utilizadas em hospitais com grande êxito (SPEAR, 2005).Neste contexto o uso de dispositivos poka-yokes apoiados na tecnologia da informação pode ser entendido como uma prática para lidar com os erros humanos, e ao mesmo tempo, melhorar a qualidade dos serviços de saúde (SHAHIN; GHASEMAGHAEI, 2010; GROUT, 2007).

A partir dessa perspectiva, este trabalho procura melhor compreender o uso de dispositivos poka-yokes como um meio de redução de erros médicos e de melhoria da qualidade de serviços de saúde. Para tanto, ele se apóia na teoria existente sobre o tema, e em um estudo de caso sobre o processo de prescrição médica em uma clínica especializada na prevenção e no tratamento de doenças neoplásicas.

\section{Referencial Teórico}

Segundo Berry e Seltman (2010) existem algumas diferenças dos serviços de saúde em relação à maioria dos outros serviços. É fato que os clientes chegam aos hospitais e clínicas com uma combinação de doenças, ferimentos, dor, incertezas e medo. Quando ficam internados nestes locais, necessitam abrir mão de parte expressiva de sua liberdade. Pode-se dizer que os serviços de saúde compartilham uma dinâmica própria, a saber:

- Seu principal benefício é intangível;

- O desempenho do serviço é muito dependente de mão de obra e talento;

- O cliente deve estar fisicamente presente para receber o serviço;

- A demanda do cliente pelo serviço não é distribuída de maneira igual;

- As necessidades e preferências dos clientes são diversas, exigindo que a organização disponha de um grande conjunto de habilidades e outros recursos que devem estar prontamente acessíveis;

- A confiabilidade do serviço e segurança é essencial;

- Diversos prestadores de serviço contribuem para a experiência do cliente demandando um esforço de grande coordenação; e 

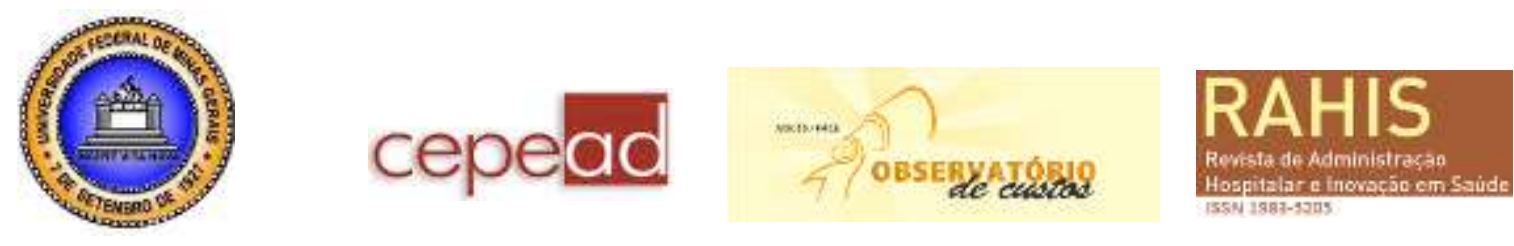

- As organizações de serviço trabalham com uma cadeia complexa, com inúmeras partes independentes.

Durante o processo de prestação de cuidados de saúde, muitos erros médicos podem ocorrer. O erro médico é tratado como o uso não intencional de um plano incorreto para alcançar um objetivo (erro de execução), ou a execução incorreta de uma ação planejada (erro de planejamento) (REASON, 1990). Para a National Cordinating Council for Medication Error Reporting and Prevention, citado por Carvalho et al. (1999, p.43), um erro médico pode ser conceituado como "qualquer evento previsível que pode ser causado ou surgir do uso inconveniente ou falta de uma medicação ou causar prejuízo ao paciente...". Ou melhor dizendo, pode ser caracterizado como um dano não intencional causado ao paciente pela intervenção assistencial, e não pela doença de base (AMAYA, 2009).

Leape et al. (1993) caracteriza quatro tipos de erros médicos que acabam resultando em algum tipo de dano para os pacientes. São eles, Erros de diagnóstico: Erros ou atraso no diagnóstico, na indicação dos testes, ao empregar testes obsoletos, ou omissão diante dos resultados apresentados nos testes realizados. Erros no tratamento: São erros na execução de uma operação, procedimento ou teste, erros na administração do tratamento, na dose da droga ou no método de usar a droga, e ainda atrasos evitáveis no tratamento ou na resposta a um teste que apontou problema. Preventiva : Inexistência de um tratamento profilático, a falta de fiscalização ou acompanhamento do tratamento. E Outros: Existem por falhas na comunicação, nos equipamento ou mesmo falha de outros sistemas existentes.

$\mathrm{O}$ erro na prescrição de um medicamento pode ser enquadrado como um tipo de erro de tratamento. Conforme Carvalho et al. (1999), o processo de administração de medicamentos com o paciente internado envolve várias fases inter-relacionadas que estão interligadas por uma equipe multifuncional. Inicia-se tal processo no momento da prescrição médica, após avaliação e diagnóstico do paciente, continuando com a transcrição da prescrição médica pelo médico, distribuição do medicamento pelo farmacêutico, preparação e administração pelo enfermeiro.

Segundo Cabral (2002) para que um medicamento seja ministrado de forma correta, o médico deve escrever a prescrição de modo correto, o farmacêutico deve avaliar se a prescrição é apropriada, e em seguida aviá-la de forma correta. O enfermeiro então deve verificar se a prescrição é adequada para em seguida administrá-la. Uma ruptura em qualquer ponto dessa cadeia pode levar a um erro médico.

Embora a maioria dos erros médicos sejam causados por erros humanos, dificilmente têm uma única causa (BUCKLEY et al., 1997; CASSIANI, 2000). É importante analisá-los como sendo uma falha do sistema a uma falha de indivíduos.

Segundo Cassiani (2000), de acordo com a American Society of Hospitall Pharmacists $(A S H P)$ existem algumas estratégias que se implementadas podem prevenir ou reduzir os erros na medicação, a saber : Prescrição médica com entrada direta no computador e envio à farmácia; Uso de código de barras no processo de administração do medicamento; Desenvolvimento de sistemas mais efetivos para monitorar e relatar eventos adversos à medicação; e Utilização de medicamentos em doses unitárias e preparo, pela farmácia, de medicamentos injetáveis por via endo-venosa, reduzindo erros de mensuração.

Uma abordagem para lidar com erros médicos é a adoção de dispositivos à prova de falhas nos serviços (GROUT, 2007). Estes dispositivos também são chamados de "PokaYokes". 

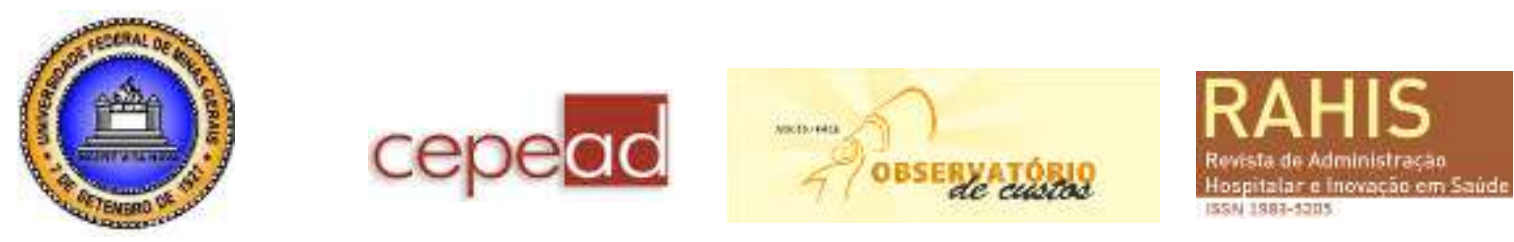

Poka-yokes são dispositivos ou mecanismos que impedem que um erro ou defeito aconteça (SHINGO, 1986). O uso de poka-yokes estabelece o redesenho de processos de modo que os erros possam ser impedidos ou imediatamente detectados e corrigidos. Eles permitem que processos aconteçam sem problemas, pois são consideradas soluções à prova de falhas (KUMAR; STEINEBACH, 2008). Além disso, seu uso é uma maneira de ajudar as pessoas a fazerem as coisas da forma correta já na primeira vez (SHINGO, 1987).

Os poka-yokes impedem que um erro inevitável se torne um defeito de serviço e seja percebido pelo cliente. Os poka-yokes partem do pressuposto de que as pessoas não cometem erros ou fazem o trabalho de modo incorreto intencionalmente, mas, por diversas razões, os erros podem ocorrer e realmente ocorrem. Portanto, o erro humano ocorre por um problema ou falha no desenho do processo (LIKER; MÉIER, 2007).

Inicialmente a aplicação de dispositivos poka-yokes concentrava-se nos processos de manufatura, sendo, portanto, orientada para produtos. Entretanto, os poka-yokes podem ser aplicados aos serviços, mas existem duas diferenças óbvias entre as operações de manufatura e serviços que devem ser consideradas nesta nova aplicação.

Em primeiro lugar, os poka-yokes devem levar em consideração que algumas atividades são realizadas pelos clientes no serviço. Isto implica que erros cometidos pelos clientes podem afetar diretamente o resultado e qualidade do serviço. A participação dos clientes nos processos de manufatura é quase inexistente. Normalmente ela ocorre após a entrega do produto e durante o seu uso. Em segundo lugar, muitos serviços envolvem múltiplas formas de interação entre a empresa e seus clientes. Essas podem ocorrer em diferentes modos de contato: face a face, por telefone, pelo correio, via Internet, por meio de equipamentos como caixas eletrônicos ou quiosques (CHASE; STEWART, 1994).

Existem varias aplicações de poka-yokes aos serviços e elas podem ser classificadas de várias formas. A primeira classificação esta relacionada se os mesmos são aplicados às atividades realizadas pelo prestador de serviços ou pelo cliente. Quando os dispositivos são aplicados nas atividades realizadas pelos clientes, eles podem ser aplicados em um dos três momentos distintos: antes da entrega do serviço; durante a entrega do serviço e, após a entrega do serviço. Quando os dispositivos são aplicados em atividades realizadas pelos prestadores de serviço, eles podem ser divididos em três Ts: relativos a Tarefa a ser realizada; relativo ao Tratamento dado ao cliente e ; finalmente relativo às características Tangíveis ou ambientais das instalações de serviços (CHASE; STEWART, 1994).

Shahin e Ghasemaghaei (2010), baseando-se em sua experiência e nos diversos pokayokes existentes classificaram os mesmos em três categorias: Poka-yokes relacionados ao layout, que utilizam o espaço físico e o local para informar, direcionar ou limitar a participação do cliente. Poka-yokes apoiados no auto-serviço, que possibilitam que os serviços sejam entregues ou obtidos no momento adequado ao cliente, o que evita a demora e atrasos. E Poka-yokes apoiados na Tecnologia, que utilizam a tecnologia para evitar falhas no processo de serviço conduzido pela empresa ou pelo cliente.

Considerando essa perspectiva, Bayers (1994) aponta que identificar os erros em sua fonte e impedir sua conversão em defeitos é o princípio fundamental por trás poka-yoke. Erro de verificação cria qualidade em processos e diminiu o retrabalho. Sendo assim, o poka-yoke é uma abordagem proativa para controle de qualidade em serviços. Segundo o autor, métodos tradicionais têm sido reativos, gerando retrabalho. Já a verificação do erro é um conceito poderoso porque é simples e eficaz.

A qualidade em serviços tornou-se uma importante estratégia corporativa para organizações de cuidados de saúde. Ela é vista como a combinação de elementos discretos 

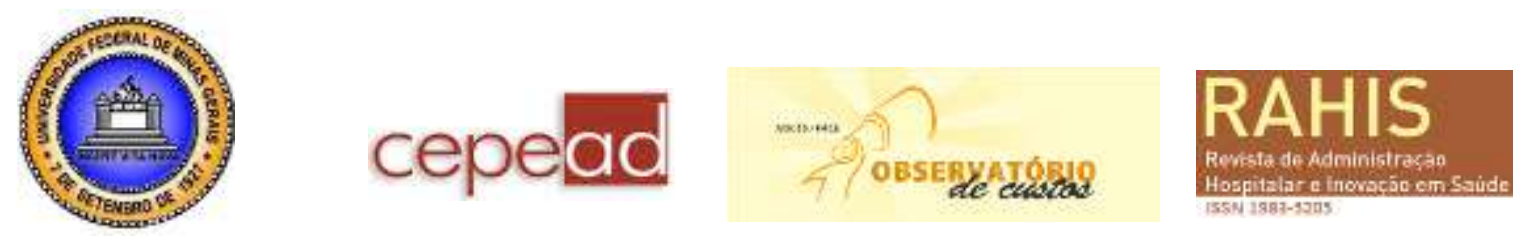

que se correlacionam com o desempenho do serviço. Os cinco elementos que compõem a qualidade de serviço são: tangíveis (instalações físicas, equipamentos, funcionários, material impresso), confiabilidade (capacidade para executar o serviço de forma precisa e confiável), capacidade de resposta (disposição para trabalhar com os clientes e fornecer o serviço de alerta), garantia (conhecimento e cortesia dos funcionários da linha de frente; sua capacidade de inspirar confiança)e empatia (PARASURAMAN; ZEITHAML; BERRY, 1985; PARASURAMAN; ZEITHAML; BERRY, 1996).

A percepção da qualidade de um serviço é a impressão sobre uma entidade de excelência total. Representa a diferença entre o esperado e o percebido em um serviço (BITNER; HUBBERT, 1994).No setor de saúde a medição da qualidade percebida pelo cliente se dá através da satisfação do paciente (LEE; KHONG; GHISTA, 2006).

A qualidade percebida em serviços de saúde pode ser avaliada com base a dimensões globais, tais como qualidade técnica, qualidade do ambiente, qualidade administrativa e qualidade interpessoal (DAGGER; SWEENEY; JOHNSON, 2007). Entretanto, existam estudos que comprovam que os mais importantes determinantes da satisfação do paciente são as relações interpessoais e seus aspectos relacionados aos cuidados (CROWEE et al., 2002).

Considerando que os poka-yokes são uma ferramenta importante no controle de qualidade e que afetam o desempenho do serviço relativo a confiabilidade, como a sua implantação pode ser percebida pelo cliente? A implantação de dispositivos a prova de falha no processo de administração de medicamentos em uma clínica de oncologia pode afetar a satisfação percebida pelo seu usuário? Esse é o objetivo desse trabalho e seu conteúdo será discutido a seguir.

\section{Metodologia}

Segundo Yin (2001, p.32) um estudo de caso é :“ uma investigação empírica que investiga um fenômeno contemporâneo dentro do seu contexto da vida real, especialmente quando os limites entre fenômeno e o contexto não estão claramente definidos". Já Hartley (1995) pontua que, o estudo de caso consiste em uma investigação detalhada que freqüentemente utiliza dados coletados durante um período de tempo, de uma ou mais organizações, ou grupos provendo uma análise de contexto e de processos envolvidos.

Considerando que o estudo em questão se refere a um estudo qualitativo como uma descrição holística e intensiva de um fenômeno bem delimitado, onde o interesse dos pesquisadores está voltado para a compreensão dos processos que ocorrem em um contexto empresarial, o estudo de caso se mostra o método mais adequado para a pesquisa em análise. Nesse sentido, a pesquisa em questão se caracteriza como pesquisa qualitativa, descritiva tendo como método o Estudo de Caso.

A unidade de análise deste estudo é o processo de administração de medicamentos de uma empresa especializada na prevenção e no tratamento das doenças neoplásicas, cujo nome fantasia é Oncomed. A escolha da empresa se deu devido a criticidade do serviço prestado pela organização e por ser uma das empresas pioneiras em Belo Horizonte em adotar o uso de dispositivos a prova de falha no processo de prescrição médica.Além disso, em razão do envelhecimento da população associado à redução de taxas de mortalidade por doenças cardiovasculares e infecciosas, as doenças neoplásicas têm aparecido com maior frequência entre os brasileiros. Com o aumento de sobrevida dos pacientes com câncer, em função de tratamentos mais eficazes, as doenças neoplásicas ganharam maior atenção nos últimos anos. 

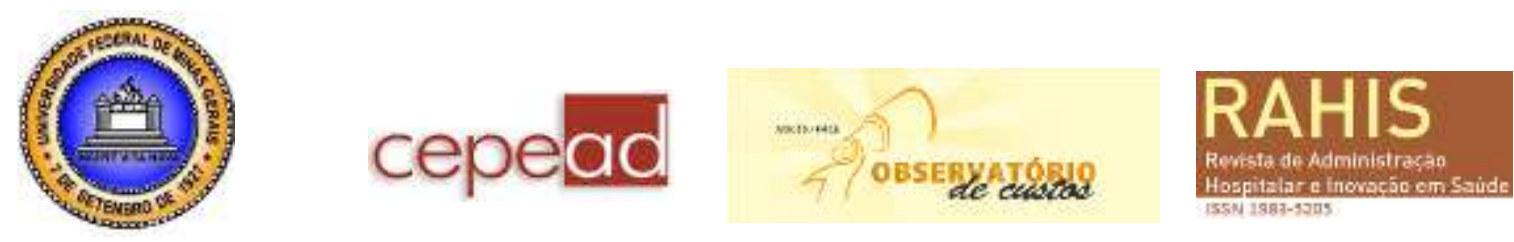

Os dados para análise foram coletados através de entrevistas não estruturadas, com profissionais responsáveis pela operação do serviço e através de relatórios de pesquisas de satisfação de clientes realizadas pela clínica no período de 2008 a 2012. Considerando que os dados da pesquisa já se encontravam tabulados, utilizou-se de ferramentas de análises estatística descritiva paramétrica e não paramétrica.

\section{Pesquisa de Campo}

A empresa analisada tem como nome fantasia Oncomed. Ela foi fundada em 20 de junho de 1994 e é especializada na prevenção e no tratamento das doenças neoplásicas. A partir de outubro de 2008 a Oncomed revisou o seu processo de atendimento a pacientes, como parte de ações voltadas para a busca da qualidade em serviços e da certificação. A partir dessa data, todo o processo de prescrição médica passou a iniciar com o protocolo padronizado por patologia.

Através de um sistema de prescrição médica acompanhada por computador, o médico seleciona o tratamento por neoplasia, acrescenta peso e altura no sistema, e o mesmo faz o cálculo da dosagem do medicamento em uma determinada sequência, com os tempos de infusão de cada medicamento. Através da parametrização de diluente de medicamentos, o sistema consegue avaliar a incompatibilidade medicamentosa da prescrição realizada, informando isso ao médico.

Em seguida, a prescrição segue para a farmácia, através de sistema computadorizado, onde o farmacêutico irá verificar a dosagem. O farmacêutico identifica os materiais e medicamentos necessários, separa-os e dá baixa ao estoque. Em seguida, se inicia o processo da diluição do soro. Quando finalizada a diluição, o soro recebe uma etiqueta com o nome do paciente, dados da medicação e o código de barras respectivo, de acordo com a sequência da prescrição. O sistema só permite o preparo do próximo medicamento quando é finalizado o processo do soro atual.

Ao chegar à enfermagem, o paciente recebe uma pulseira com o seu nome e com a numeração da prescrição através de um código de barras. A enfermagem recebe o soro preparado pela farmácia com a numeração do código de barra na sequência da prescrição. A partir de então se inicia a administração da medicação.

A aplicação da medicação é feita com o apoio de aparelhos que leêm códigos de barras, aparelhos palmtop. O enfermeiro passa o aparelho no código de barras do próprio crachá e em seguida passa o aparelho palmtop na pulseira do paciente aguardando uma mensagem com o nome do paciente e o medicamento a ser ministrado. Logo após, o enfermeiro passa o palmtop no código de barras do soro e aguarda a mensagem "medicamento checado com sucesso". Caso não seja o medicamento correto, aparecerá no visor do palmtop a mensagem "este medicamento não confere com a seqüência da ordem" ou "erro: o número do medicamento não é igual ao da pulseira". O aparelho alerta o erro ao técnico de enfermagem, registra-o e somente após a verificação e correção do procedimento permite o prosseguimento do processo. $\mathrm{O}$ erro não acontece, mas o palmtop registra a possibilidade de sua ocorrência.

Ao finalizar a medicação, o enfermeiro passa o palmtop na pulseira do paciente para verificar se todos os soros foram ministrados. Em caso positivo, o paciente é liberado.

Segundo abordagem de Chase e Stewart, o primeiro passo na verificação de prováveis falhas é rever cada etapa do processo de serviço e identificar onde e quando ocorrem as 

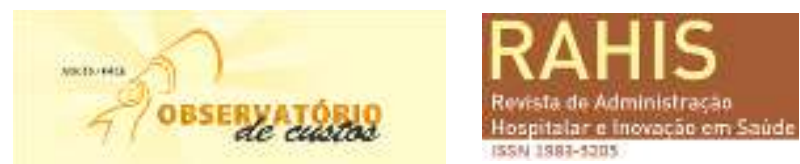

falhas. A partir de um macrofluxo de processo, com etapas de processamento e fluxo das informações, é possível definir uma linha de visibilidade, onde aparecem as etapas do processo em que os clientes podem ver os defeitos e uma linha de interação, que é onde ocorrem as entregas entre o cliente e o prestador de serviço. A partir dessa macrofluxo é possível identificar os prováveis erros, de modo que os mesmos sejam bloqueados, antes de se transformarem em defeitos.

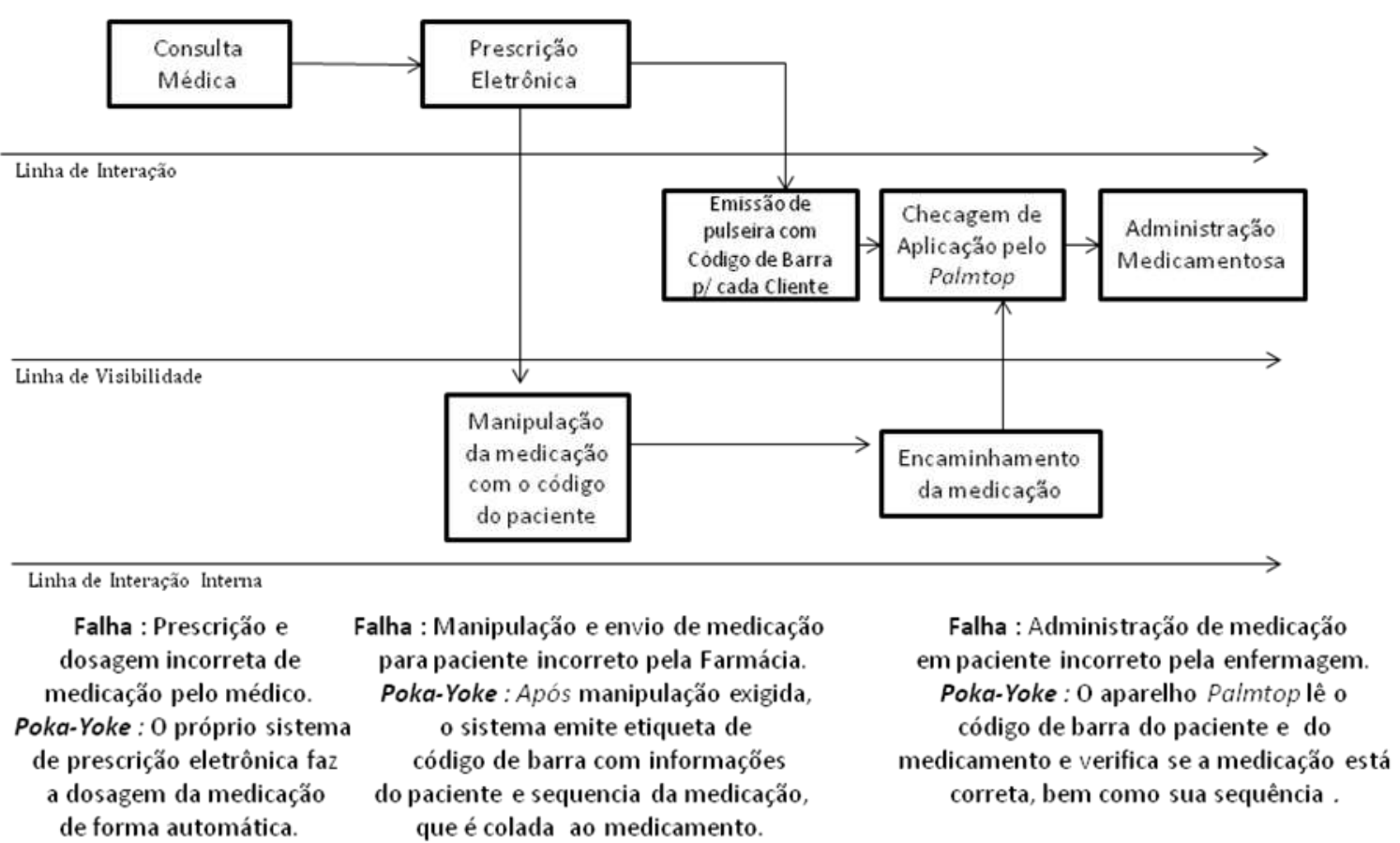

Figura 1: Diagrama de Serviço de atendimento da Oncomed - Elaborado pelos autores

Com base na abordagem de Chase e Stewart (1994) e na descrição do processo de prescrição médica da Oncomed apresentado anteriormente, foi possível gerar um macrofluxo dos processos e informações, apresentado na Figura 1 e verificar quais os dispositivos a prova de falha ou poka-yokes foram introduzidos no processo de prescrição médica da clínica, evitando a presença do erro ou defeito.

Os poka-yokes introduzidos no processo de prescrição médica na clínica analisada são caracterizados como relativos à tarefa e apoiados por tecnologia. O palmtop registra os erros que são identificados, erros esses tanto de alteração de ordem prescrita na administração, quanto de troca de medicamentos entre pacientes. O aparelho alerta o erro ao técnico de enfermagem, registra-o e somente após a verificação e correção do procedimento permite o prosseguimento do processo. $\mathrm{O}$ erro não acontece, mas o palmtop registra a possibilidade de sua ocorrência. Todo o processo de administração de medicamentos é rastreado pelo aparelho palmtop, que indica também o número total de pacientes atendidos, por técnicos de enfermagem, bem como o índice de erros detectados por funcionários.

$\mathrm{O}$ aparelho palmtop foi introduzido na empresa pesquisada a partir do $2^{\circ}$ semestre de 2008. Com a introdução do dispositivo, a clínica passou a coletar dados referentes ao número de checagens e de erros prováveis detectados a partir de 2010, números antes não conhecidos. Os mesmos são apresentados a seguir. 


\section{cepead}
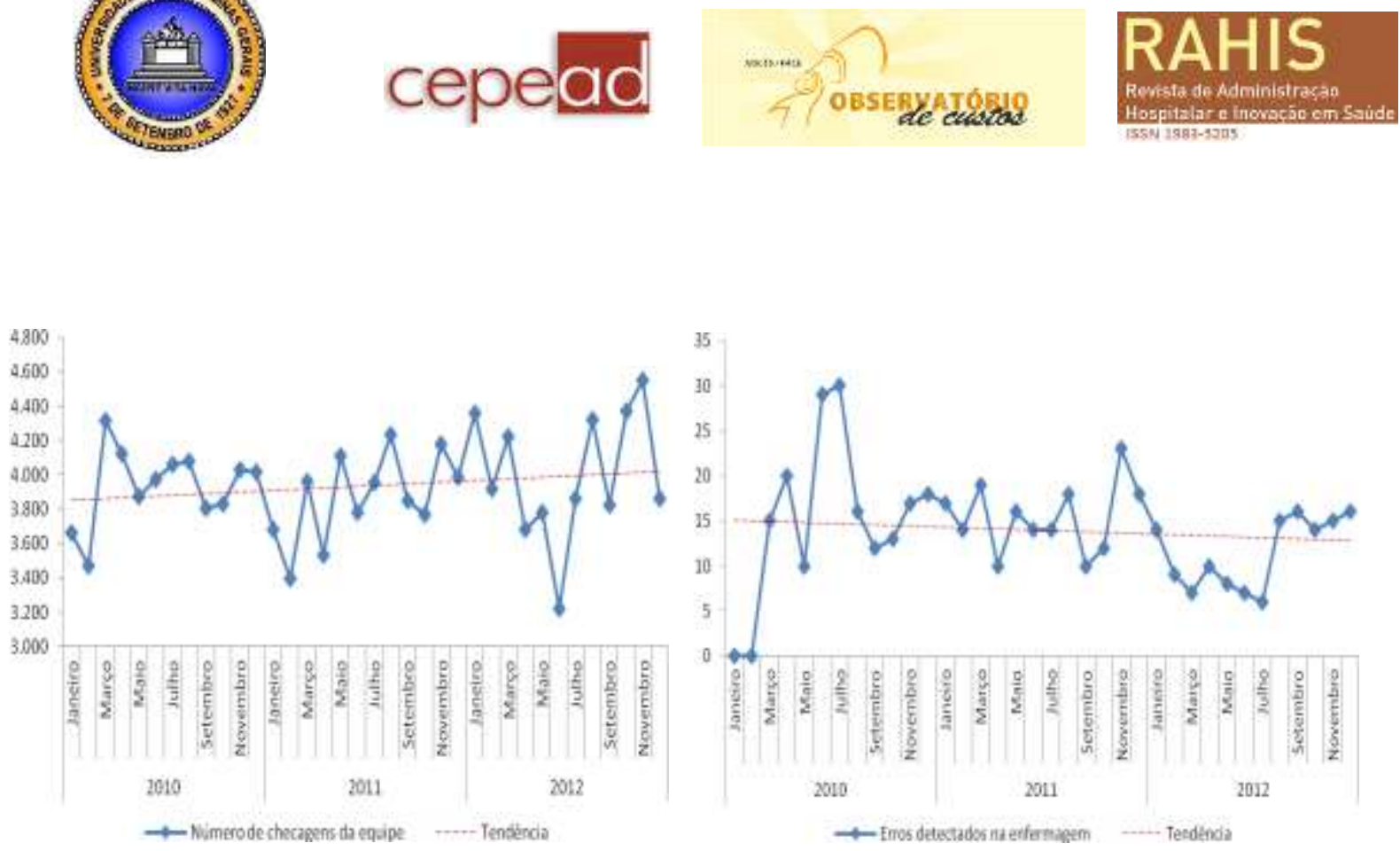

Figura 2 - Número de checagens X Número de erros detectados na enfermagem no período de janeiro de 2010 a dezembro de 2012. Oncomed/Belo Horizonte.

Com base nos graficos acima, percebe-se uma tendência de decréscimo no número de erros verificados através do palmtop, embora o número de checagens tenha aumentado, tendo em vista que o número de clientes da clínica aumentou nos ultimos anos.

Com o passar do tempo, o número de prováveis erros registrados pelo palmtop reduziu. Ao avaliar numericamente o relacionamento entre o tempo, o número de checagens e o número de erros detectados, utilizando a técnica de análise de regressão linear múltipla, onde número de erros é considerado a variável dependente, o tempo apresenta valor p inferior a $0,05(-0,42)$. Esse valor demonstra uma relação negativa com os erros de checagem, indicando que quanto mais o tempo passa, mais o número de erros detectados diminui . Por outro lado, existe uma relação positiva entre o número de checagens e o número de erros detectados, com coeficiente igual a 0,51 .

Já na análise de regressão, com coeficiente de determinação $\mathrm{R}^{2}$ ajustado, que minimiza a influência do tamanho da amostra, o valor encontrado 0,225 indica baixo poder de explicação do modelo. Isso significa que o tempo e o número de checagens explicam pouco a variabilidade no número de erros. Ou seja, os erros são explicados também por outros fatores.

Dessa forma, constata-se que embora a clínica adote ferramentas de gestão da qualidade, através de maior conscientização de sua equipe de enfermagem, e exista uma curva de aprendizagem quanto a tarefa realizada, com o passar do tempo, a provável ocorrência do erro permanece em menor proporção, se mostrando inerente ao sistema.

Em contrapartida, um aspecto importante observado é que o componente confiança, bem como outras qualidades de carater interpessoal, apresentam um peso importante na prestação do serviço de saúde, sob a perspectiva do cliente da clínica.

Como apresentado na tabela que segue, segundo clientes da Oncomed a confiança é o segundo quesito mais importante para uma clínica de doenças neoplásicas, perdendo apenas para o atendimento personalizado. Além disso, a confiança já é um valor percebido pelos seus 


\section{cepese}

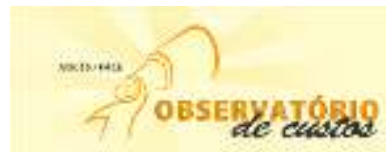

usuários. Como a clínica já pratica a confiança como um valor importante, essa é uma das razões que faz novos clientes procurarem a clínica.

\begin{tabular}{|c|c|c|c|c|c|}
\hline \multicolumn{2}{|c|}{$\begin{array}{l}\text { O que é importante para uma } \\
\text { clinica como a Oncomed? }\end{array}$} & \multicolumn{2}{|c|}{$\begin{array}{l}\text { Valores e Princípios } \\
\text { percebidos na Oncomed? }\end{array}$} & \multicolumn{2}{|l|}{ Porque escolheu a Oncomed ? } \\
\hline $\begin{array}{l}\text { Atendimento } \\
\text { diferenciado }\end{array}$ & $24,03 \%$ & Respeito & $40,44 \%$ & Indicação & $37 \%$ \\
\hline Confiança & $20,13 \%$ & Carinho & $16,91 \%$ & médica & $18 \%$ \\
\hline Tratamento diferenciado & $19,48 \%$ & Confiança & $13,24 \%$ & Acolhimento & $12 \%$ \\
\hline Respeito & $18,18 \%$ & Acolhimento & $13,24 \%$ & Confiança & $13 \%$ \\
\hline Acolhimento & $11,69 \%$ & Humanização & $13,24 \%$ & Tratado de forma especial & $13 \%$ \\
\hline Humanização & $10,39 \%$ & Dedicação & $12,50 \%$ & Possui melhores instalações & $7 \%$ \\
\hline
\end{tabular}

Tabela 1 - Aspectos importantes para um cliente de uma clinica de doenças noeplásicas Oncomed/Belo Horizonte, 2001.

Percebe-se que, em função da natureza do tratamento oncológico, em que o sequenciamento da medicação se mostra importante, a confiança depositada no agente responsável pela administração do medicamento é significativa. Além disso, como os pacientes estão mais fragilizados psicologicamente, necessitam uma maior atenção e satisfação com aspectos relacionados à humanização e ao relacionamento interpessoal.

No que diz respeito à qualidade percebida pelos usuários, a clínica realiza desde 2008 pesquisas semestrais junto a seus pacientes e acompanhantes através de questionário. Com uma amostra de 250 questionários, em média, estimada com base no número de pacientes atendidos no período de seis meses anteriores à realização da pesquisa, os participantes respondem às alternativas atribuindo notas em uma escala de cinco pontos (modelo Lickert).

Os quesitos avaliados pelo questionário são: atendimento na portaria, atendimento na recepcção, atendimento na enfermagem, estrutura do ambiente, limpeza, atendimento telefônico, equipe médica, recursos de distração, atendimento da psicóloga e atendimento da nutricionista. Quanto ao serviço de enfermagem, onde se concretiza o processo de administração de medicamentos, os quesitos avaliados são: confiança, respeito, paciência e bom humor. A partir dos dados já tabulados disponíveis na empresa, a tabela que segue mostra o nível de satisfação dos clientes para alguns quesitos, no período anterior e posterior à introdução dos poka-yokes no processo de administração de medicamentos.

\begin{tabular}{|c|c|c|c|c|c|c|c|c|c|}
\hline $\begin{array}{l}\text { Indice de } \\
\text { Satisfação }\end{array}$ & $\begin{array}{l}1^{\circ} . \text { Sem } \\
2008\end{array}$ & $\begin{array}{l}2^{\circ} . \text { Sem } \\
2008\end{array}$ & $\begin{array}{l}1^{\circ} \text { Sem } \\
2009\end{array}$ & $\begin{array}{l}2^{\circ} \text { Sem } \\
2009\end{array}$ & $\begin{array}{l}1^{\circ} \text { Sem } \\
2010\end{array}$ & $\begin{array}{l}2^{\circ} \text { Sem } \\
2010\end{array}$ & $\begin{array}{l}1^{\circ} . \text { Sem } \\
2011\end{array}$ & $\begin{array}{l}2^{\circ} \text { Sem } \\
2011\end{array}$ & $\begin{array}{l}1^{\circ} \mathrm{Sem} \\
2012\end{array}$ \\
\hline Global & 81,05 & 89,16 & 88,45 & 92,81 & 91,39 & 91,77 & 86,30 & 86,88 & 97,27 \\
\hline Enfermagem & 86,18 & 89,77 & 90,42 & 94,24 & 94,65 & 92,60 & 85,62 & 89,99 & 98,72 \\
\hline Confiança & 86,02 & 91,61 & 92,10 & 96,09 & 97,56 & 89,00 & 87,60 & 88,60 & 97,90 \\
\hline Respeito & 88,65 & 92,95 & 95,24 & 97,79 & 96,93 & 94,92 & 89,11 & 91,04 & 98,13 \\
\hline Paciência & 85,95 & 90,39 & 91,58 & 95,61 & 95,71 & 93,71 & 87,07 & 90,91 & 98,60 \\
\hline Bom Humor & 86,96 & 88,96 & 89,47 & 95,57 & 93,83 & 94,29 & 89,72 & 90,30 & 98,60 \\
\hline
\end{tabular}

Tabela 2: Índice de Satisfação do cliente- Oncomed/Belo Horizonte, 2008/2012. 

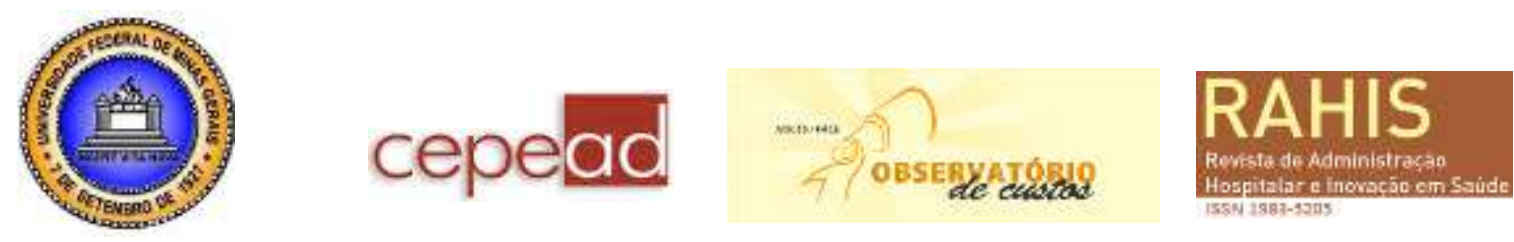

Para avaliar se houve melhoria dos índices de satisfação a partir da introdução dos dispositivos a prova de falha, utilizou-se a técnica de comparação das estimativas e dos intervalos de confiança dos grupos de índices de satisfação antes e depois. Ao se agrupar os estudos até 2008, com amostra de 484 respondentes, a margem de erro encontrada foi de 2,3 pontos percentuais. No agrupamento dos estudos após 2008 com amostra de 1577 respondentes, a margem de erro encontrada foi de 1,4 pontos percentuais.

Nas duas situações, utilizou $\mathrm{p}=0,9$ para os cálculos, ao verificar os dados históricos. As estimativas foram calculadas a partir do número de respostas de nível 4 e 5 da escala Linkert no período, em relação ao tamanho da amostra. Levando-se em consideração a margem de erro dos estudos agrupados por período, obteve-se os limites inferiores das estimativas (estimativa - margem de erro) e os limites superiores (estimativa + margem de erro).

\begin{tabular}{|c|c|c|c|}
\hline \multicolumn{2}{|c|}{ Indice de Satisfação } & \multirow{2}{*}{ Até $2^{\circ}$.Sem 2008} & Após $2^{\circ}$. Sem 2008 \\
\hline Margem de erro * & & & $1,4 \%$ \\
\hline \multirow[t]{3}{*}{ Global } & Limite Inferior & $82,2 \%$ & $\underline{89,5 \%}$ \\
\hline & Estimativa & $84,5 \%$ & $\overline{90,9 \%}$ \\
\hline & Limite Superior & $86,8 \%$ & $92,3 \%$ \\
\hline \multirow[t]{3}{*}{ Enfermagem } & Limite Inferior & $85,4 \%$ & $\underline{92,1 \%}$ \\
\hline & Estimativa & $87,7 \%$ & $93,5 \%$ \\
\hline & Limite Superior & $90,0 \%$ & $94,9 \%$ \\
\hline \multirow[t]{3}{*}{ Confiança } & Limite Inferior & $86,3 \%$ & $91,9 \%$ \\
\hline & Estimativa & $88,6 \%$ & $93,3 \%$ \\
\hline & Limite Superior & $\underline{90,9 \%}$ & $94,7 \%$ \\
\hline \multirow[t]{3}{*}{ Respeito } & Limite Inferior & $88,3 \%$ & $\underline{93,9 \%}$ \\
\hline & Estimativa & $90,6 \%$ & $95,3 \%$ \\
\hline & Limite Superior & $92,9 \%$ & $96,7 \%$ \\
\hline \multirow[t]{3}{*}{ Paciência } & Limite Inferior & $\overline{85,7 \%}$ & $\underline{92,4 \%}$ \\
\hline & Estimativa & $88,0 \%$ & $\overline{93,8 \%}$ \\
\hline & Limite Superior & $\underline{90,3 \%}$ & $95,2 \%$ \\
\hline \multirow[t]{3}{*}{ Bom Humor } & Limite Inferior & $\overline{84,8 \%}$ & $92,0 \%$ \\
\hline & Estimativa & $87,1 \%$ & $\overline{93,4 \%}$ \\
\hline & Limite Superior & $89,4 \%$ & $94,8 \%$ \\
\hline
\end{tabular}

Tabela 3: Comparativo de Estimativas de Satisfação no período de 2008 a 2012. Oncomed/Belo Horizonte, 2013.

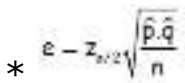

Como visto na tabela 3, todos os índices após 2008 são superiores aos índices anteriores, visto que o limite inferior das estimativas após 2008 são superiores aos limites superiores do período até 2008. Sendo assim, pode-se afirmar que com a introdução dos pokayokes, houve melhoria no índice de satisfação global do serviço de saúde da Oncomed, bem como no índice de satisfação com a enfermagem e as dimensões que compoe o serviço de enfermagem.

Para avaliar se existe um relacionamento entre a eliminação dos erros na administração de medicamentos com os índices de satisfação torna-se necessário calcular a taxa de erros ocorrida no período avaliado. Entretanto, os prováveis erros detectados pelo uso do palmtop começaram a ser registrados apenas a partir de 2010. Como existem apenas 5 

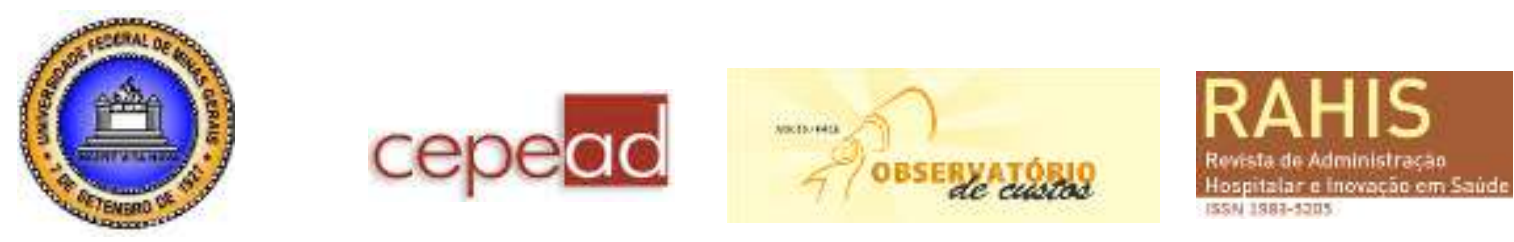

medições nesse período, tornando a amostra pequena para os métodos que exigem normalidade na distribuição de probabilidade associada (testes paramétricos), foram usados testes não-paramétricos. Sendo assim, para avaliar o relacionamento entre a taxa dos erros detectados pelo palmtop e os índices de satisfação utilizou-se o coeficiente de correlação de Spearman.

O índice de correlação de Spearman é uma medida que varia de -1 a 1 e indica o grau e o sentido do relacionamento linear entre duas variáveis. Valores próximos de -1 indica alta correlação negativa, valores próximos de zero indicam ausência de correlação e valores próximos de 1 indicam alta correlação positiva( LEVIN, 1987).

Foi realizado também um teste de hipóteses para verificar se tal correlação é significativamente diferente de zero. Como resposta de tal teste de hipótese obteve-se o valor p, indicando a existência de correlação significativa ao nível de 5\%.

Como resultado, obteve-se um coeficiente de correlação de $-0,671$ para o índice de satisfação global com um valor $\mathrm{p}$ de 0,215 . Para o nível de satisfação com a enfermagem obteve-se um coeficiente de correlação de $-0,894$ e um valor p de 0,041 . Para os índices que compoem o atendimento da enfermagem, confiança, respeito, paciência e bom humor os coeficientes de correlação encontrados foram $-0,894 ;-0,894 ;-0,894$ e $-0,671$ e os valores $p$ 0,$041 ; 0,041 ; 0,041$ e 0,215 respectivamente.

Os valores negativos encontrados indicam que existe uma correlação negativa entre a taxa de erros e o índice de satisfação, ou seja, quanto maior a taxa de erros, menor o índice de satisfação. Entretanto, é importante também considerar o valor p, que deve estar abaixo de 0,05. Diante disso, considera-se que o índice de satisfação com a enfermagem reflete a eliminação de erros ocorrida no processo de administração de medicamentos, bem como a confiança, o respeito e a paciência.

Sendo assim, percebe-se que o uso de dispositivos a prova de falha favorecem a satisfação dos clientes em dimensões não apenas relacionadas à confiabilidade, mas a aspectos do serviço de administração de medicamentos como um todo, à aspectos ligados aos relacionamentos interpessoais.

Ao verificar a tabela 1 apresentada anteriormente, dimensões de qualidade interpessoal e do cuidado são importantes para os clientes da clínica pesquisada. Isso corrobora com as pesquisas da área de qualidade em serviços. Ao considerar esses aspectos como sendo os mais importantes sob o ponto de vista do cliente, o uso de dispositivos poka-yokes se mostram uma ferramenta eficiente na prestação do serviço de saúde.

\section{Conclusão}

De acordo com Jonhston (2008), os serviços são definidos como uma combinação de resultados e experiência entregues e recebidos pelos clientes. O processo de prescrição médica envolve um conjunto de subsistemas, criando oportunidades para o surgimento de erros. Além disso, o fato gerador do erro, muitas vezes, pode estar presente em mais de um subsistema. Sendo assim faz-se necessário uma revisão de processos de modo a tornar dificil o surgimento do erro e fácil a execução da ação de forma correta.

Nesse sentido, existem na literatura de gestão dispositivos conhecidos como pokayokes, que eliminam a possibilidade do erro, determinando que as ações sejam executadas conforme o planejado. segundo Shahin e Ghasemaghaei (2010), o uso de dispositivos pokayokes apoiados na tecnologia de informação além de reduzir erros e retrabalho, auxiliam na melhoria da qualidade e conseqüentemente no aumento de produtividade em serviços. 

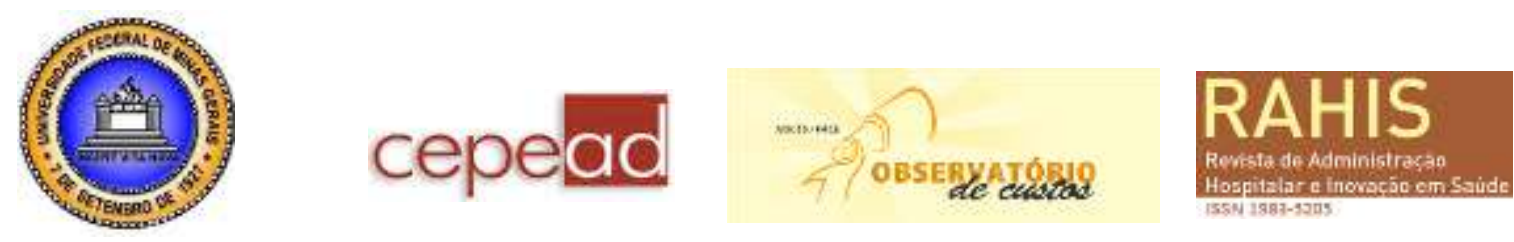

No caso específico da clínica pesquisada constatou-se que o erro é inerente ao sistema e que a introdução de dispositivos a prova de falha aumentaram a confiabilidade do processo de prescrição médica. Além disso, verificou-se que a confiabilidade é um atributo de qualidade importante para o paciente de uma clínica de tratamento oncológico. Entretanto, com a introdução dos poka-yokes, houve melhora também, na percepção e satisfação dos pacientes, com as dimensões de qualidade atreladas a cuidados e aos relacionamentos interpessoais do serviço de saúde prestado. Sendo assim, a implantação de dispositivos pokayokes no processo de prescrição médica da Oncomed trouxe impactos positivos para a percepção da qualidade do serviço de saúde da clínica.

O porquê de processos mais confiáveis afetarem positivamente dimensões de serviços atreladas a aspectos interpessoais não foi objeto desse estudo. Todavia, sugere-se para novos estudos a avaliação do impacto do uso de poka-yokes no serviço de saúde sob a perspectiva do profissional de saúde, de modo a verificar como ambientes não susceptíveis a erros afetam o desempenho dos profissionais envolvidos no processo.

\section{Referências}

AMAYA, S. L. Seguridad del Paciente: Conceptos e análisis de eventos adversos. Centro de Gestión Hospitalaria Via SALUD, n.48, p.6-21, jul 2009.

BAYERS, P.C.; Using Poka Yoke (Mistake Proofing Devices) to Ensure Quality Applied Power Electronics Conference and Exposition, APEC '94. Conference Proceedings, 1994.

BERRY, L.L.;SELTMAN, K. D. Lições de Gestão da Clínica Mayo: por dentro de uma das mais admiradas organizações de serviços do mundo, Porto Alegre: editora Bookman, 2010 .

BITNER, M.J.; HUBBERT, A.R. Encounter Satisfaction Versus Overall Satisfaction Versus Qualit. In: Rust TR, Oliver RL. Service Quality: New Directions in Theory and Practice. Thousand Oaks; 241-268, 1994.

BUCKLEY, T. A et al. Critical incident reporting in the intensive care unit Anaesthesia Vol. 52, No 5, p. 403-409, Maio,1997.

CABRAL, I. E. Administração de medicamentos. Rio de Janeiro: Reichmann \& Affonso . Editores, 2002. p.456.

CARVALHO, V.T. et al. Erros mais comuns e fatores de risco na administração medicamentos em unidades básicas de saúde. Revista Latino-Americana de Enfermagem. v.7, n.5, p.67-75, 1999.

CASSIANI, S. H. de B. Erros na Medicação: Estratégias de prevenção - Revista Brasileira de Enfermagem. V.53, no. 3 páginas 424-430, 2000.

CHASE, R.B.; STEWART, D. M. Make Your Service Fail-Safe. Sloan Management Review, Vol. 35, No 3, p. 35-44, 1994. 

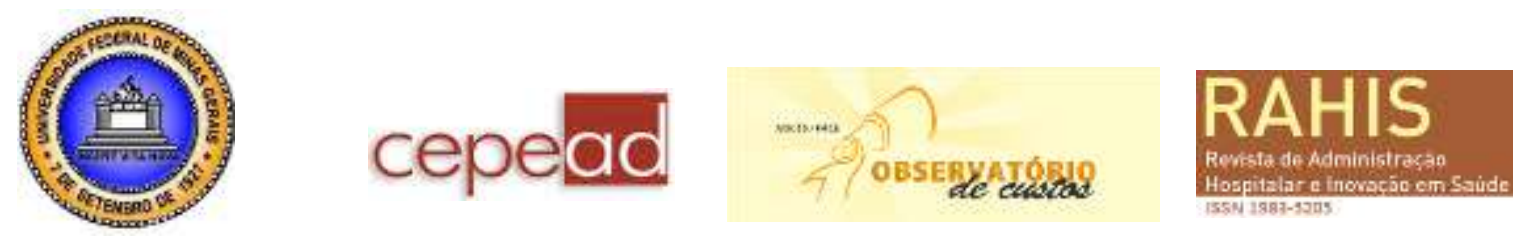

COLI, R. C. P.; ANJOS, M. F. dos; PEREIRA, L. L. Postura dos enfermeiros de uma unidade de terapia intensiva frente ao erro: uma abordagem à luz dos referenciais bioéticos. Rev. Latino-Am. Enfermagem [online]. 2010, vol.18, n.3, pp. 324-330. ISSN 0104-1169.

CROWE, R.; GAGE, H.; HAMPSON S.; HART J, KIMBER A, STOREY, L. et al. The measurement of satisfaction with healthcare: implications for practice from a systematic review of the literature. Health Technology Assessment. 6(32): 1-244, 2002.

DAGGER, T.S.; SWEENEY J.C.; JOHNSON, L.W. A hierarchical model of health service quality: scale development and investigation of an integrated model. Journal of Service Research. 10(2): 123-42, 2007.

GROUT, J. Mistake-Proofing the Design of Health Care Processes, Agency for Healthcare Research and Quality, 2007.

HARTLEY, J.F. Case studies in organizational research. In: CASSEL, C. e SYMON, G. Qualitative methods in organizational research : a practical guide, London : Sage, 1995.

JOHNSTON, R.; CLARK, G. Introduction to service operations management, In.: Johnston, Robert; Clark, Graham; Service Operations Management: improving service delivery, Pearson Education Limited, p.3-38, 2008.

KUMAR, S.; STEINEBACH, M. Eliminating US hospital medical errors. International Journal of HealthCare Quality Assurance, 21(5): 444-471, 2008.

LEAPE, L. et al. Pre-venting Medical Injury. Qual Rev Bull, Vol. 9, No 5, p.144-149, 1993.

LEE, P.M.; KHONG P.; GHISTA, D.N. Impact of deficient healthcare service quality. The TQM Magazine. 18(6): 563-71, 2006.

LEVIN J. Estatística Aplicada a Ciências Humanas. São Paulo: Harbra; 1987.

LIKER, J. K.; MEIER, D. O Modelo Toyata: manual de aplicação, Porto Alegre. Bookman, 2007.

O'CONNOR, E.; COATES, H.M.; YARDLEY, I.E.; WU, A.W. Disclosure of Patient Safety Incidents: A Comprehensive Review. International Journal for Quality in Health Care, v.22, n.5, p. 371-79, 2010.

PARASURAMAN, A.; ZEITHAML V.A.; BERRY, L.L. A conceptual model of service quality and its implications for future research. Journal of Marketing. 49(4): 41-50, 1985.

PARASURAMAN, A.; ZEITHAML, V.A.; BERRY, L.L. The Behavioral Consequences of Service Quality. Journal of Marketing. 60(4): 31-46, 1996.

REASON, J. Human error. New York: Cambridge University Press; 1990.

SHAHIN, A.; GHASEMAGHAEI, M. Service Poka Yoke, International Journal of Marketing Studies, Vol. 2, No. 2; November 2010. 


\section{cepead}

SHINGO, S. Zero Quality Control: Source Inspection and the Poka-Yoke System. Portland: Productivity Press; 1986.

SHINGO, S. The Sayings of Shigeo Shingo: Key Strategies for Plant Improvement. Portland: Productivity Press; 1987.

SPEAR, S.J. Fixing Health Care from the Inside, Today, Harvard Business Review, Vol. 83, No 9, 2005.

STEENHUYSEN, J. Mistakes Common in U.S. Hospitals. Reuters Health Information, v.1, 2011.

YIN, R.K. Estudo de Caso : Planejamento e métodos. Porto Alegre. Bookman, 2001. 